\title{
The Role of Technology in Adherence to Physical Activity Programs in Patients with Chronic Diseases Experiencing Fatigue: a Systematic Review
}

Andrea Albergoni ${ }^{1,2}$, Florentina J. Hettinga ${ }^{3,4}$, Antonio La Torre ${ }^{1,5}$, Matteo Bonato ${ }^{5}$ and Francesco Sartor ${ }^{2,3,6,7^{*}}$ (D)

\begin{abstract}
Background: The beneficial role of physical activity (PA) to manage the health condition of patients with chronic diseases is well known. However, adherence to PA guidelines in this group is still low. Monitoring and user-interface technology could represent a significant tool to increase exercise adherence to those particular groups who experience difficulties in adhering to regular and substantial physical activity, and could be supportive in increasing the success of PA programs and interventions. This systematic review aimed at evaluating the effect of physical activity monitoring technology in improving adherence to a PA program in patients with chronic diseases experiencing fatigue.
\end{abstract}

Methods: This systematic review was conducted according to PRISMA guidelines. The literature search was performed in Embase, Medline, Biosis, Scopus, and SPORTDiscus. We filtered the literature according to the question: "Does monitoring technology affect adherence to physical activity and exercise programs in patients with chronic diseases perceiving fatigue?".

Results: The search resulted in 1790 hits; finally, eight studies were included, with a total number of 205 patients. Study quality was moderate except for one study of high quality. Only three disease types emerged, COPD, HF, and cancer. PA programs were rather short (from 8 to 13 weeks) except for one 3-year-long study. Five studies employed pedometers and two an activity monitor. Three studies based their adherence on steps, the remaining studies focused on active minutes. Adherence was explicitly reported in two studies, and otherwise derived. Four studies showed high adherence levels (85\% week-10, 89\% week-8, 81\% week-13, 105\% week-13, 83\% average week-1-12) and three low levels (56\% week-12, 41\% year-2, 14 year-3).

Conclusion: The small number of studies identified did not allow to establish whether the use of monitoring technology could improve adherence to PA programs in patients with chronic diseases experiencing fatigue, but the current evidence seems to suggest that this is a field warranting further study, particularly into how monitoring technology can help to engage patients to adhere to PA programs.

Keywords: Guidelines, Patients, Technology, Wearables, Pedometers, Activity monitors, Cancer, COPD, Self-efficacy steps

\footnotetext{
* Correspondence: francesco.sartor@philips.com

2Department of Patient Care \& Measurements, Philips Research, Eindhoven,

The Netherlands

${ }^{3}$ School of Sport Rehabilitation and Exercise Sciences, University of Essex,

Colchester, UK

Full list of author information is available at the end of the article
} 


\section{Key Points}

- Although monitoring technology is a clear emerging trend in promoting physical activity in patients with chronic diseases and has potential, hitherto there is not enough evidence to clarify if the use of technology supports patients with chronic diseases to increase exercise adherence.

- Technology is mainly used to monitor physical activity, but not yet to improve exercise adherence. In the few studies where this was the case, adherence levels were high. The role of fatigue needs to be further researched and the definition of adherence needs to be standardized.

\section{Background}

There is a large body of evidence showing the benefit of physical activity (PA) and physical exercise for patients with chronic diseases [1]. The concept "exercise is medicine" as defined by the American College of Sports Medicine (ACSM), has been widely accepted for the prevention, and in some specific cases, for the treatment of chronic diseases such as cancer, type 2 diabetes mellitus (T2DM), cardiovascular diseases [2], and of people with disabilities [3]. In fact, PA and physical exercise have been considered a real "polypill" in primary as well as secondary prevention $[4,5]$. Since physical exercise can be considered a subcategory of PA [1], we will here include physical exercise in PA. Leisure-time activity, low, moderate, and vigorous activities have been linked to a reduction in the risk of T2DM [6], and in inflammatory markers in breast cancer survivors [7]. In cancer survivors, PA improves quality of life, cardiorespiratory fitness and strength, and it alleviates fatigue $[8,9]$. In chronic obstructive pulmonary disease (COPD) patients, PA is associated with better respiratory parameters (e.g., $\mathrm{FEV}_{1}$, dyspnea) [10]. PA has a strong effect in reducing atherosclerotic factors, typical of cardiovascular diseases [11]. Moderate-to-vigorous PA bouts were associated with lower severity of pain and fatigue in women with fibromyalgia [12], and graded exercise therapy has shown benefits for myalgic encephalomyelitis patients [13]. According to a recent meta-analysis, exercise rehabilitation improved exercise capacity as well as healthrelated quality of life of heart failure patients and it should be offered to all heart failure patients [14]. In patients with chronic psychological disorders, PA helps to increase self-esteem and to reduce depression [15].

Despite the fact that this evidence shows the importance of PA in preventing and treating patients with chronic diseases, the adherence to guidelines is still rather low. Patients with a serious mental illness were less active than the general population [15], only the $9 \%$ of them reached the PA guidelines [16]. Hartman et al. (2010) found that in Sweden, $84 \%$ of COPD, $74 \%$ of rheumatoid arthritis, $72 \%$ of T2DM and, $60 \%$ of healthy individuals did not adhere to PA guidelines. In a UK cohort of seniors, only $15 \%$ of men and $10 \%$ of women met guidelines [17] and dramatically less than $5 \%$ of adults in the USA [18].

The term adherence when referring to PA is not always well defined and uniform. Adherence to a program can be intended as the number of sessions conducted over the total number of sessions prescribed, either accounting for, or regardless whether the sessions were fulfilled or not [19]. For instance, a patient exercising three times a week, $30 \mathrm{~min}$ per session, as prescribed, would have an adherence of $100 \%$ as well as a patient training three times a week but only for $15 \mathrm{~min}$ per session, if only the number of sessions performed were to be accounted for.

The major factors that hinder exercise adherence in patients with chronic diseases are low self-efficacy, depression, anxiety, helplessness, poor social support or activity, greater perceived number of barriers to exercise, and increased pain levels during exercise [20]. In addition to these, fatigue in patients with chronic diseases is a common symptom that decreases adherence to a PA program [21]. In patients with chronic diseases, fatigue, defined as "a subjective feeling of tiredness, weakness or lack of energy" [22], is reported to be a major obstacle to PA execution [23]. Indeed, fatigue is an important factor related with low levels of PA in COPD patients [24]. Fatigue is a typical symptom in cancer patients [25] and it persists even after chemotherapy [9]. Fatigue is also a common symptom in T2DM patients [26], and PA has been shown to help to manage it [27]. Muscular pain and fatigue make PA intolerable in many chronic heart failure (HF) patients [28]. Recently, it has been found that activity pacing, a strategy to divide one's daily activities into more manageable portions, might have sustained beneficial effects on management and reduction of fatigue in persons with disabilities or chronic diseases associated with fatigue complaints [29].

In the past years, there has been a large number of strategies aiming at lowering the barriers to engage in PA programs. It is now common for patients to discuss their PA habits with their health professionals [30]. Behavioral interventions such as motivational interviewing and goal-setting are commonly used [31]. Furthermore, large-scale strategies, such as national walking programs, have been also deployed to increase PA in the entire population [32]. Recently, programs also started targeting persons with disabilities and chronic diseases for example in the ReSpAct study in the Netherlands [33]. Finally, health professional supervision by itself can increase adherence, and monitoring is its key element [34].

Electronic devices can be adopted to monitor adherence as long as they are used in a systematic manner [34]. However, there is a large gap between self-reported PA and measured PA [35] as self-reported PA is known to be 
not always reliable. For this reason, PA programs better be implemented with the use of human interface and monitoring technology such as web sites [30, 32, 36], mobile devices [37], apps [38], and wearable devices [39]. Additionally, the presence of an objective goal (daily steps or exercise minutes) can be a key-factor to successfully increase PA, without it the improvements may be limited or even absent [40]. Wearable technology was identified as the leading fitness trend in 2019 [41]. Indeed, ACSM-certified professionals identified it as a tool to positively change PA behavior [41]. Activity and exercise metrics such as step counts, distance covered, active or walking time can be tracked by pedometers, in addition, more recently, energy expenditure, activity types, and intensity can be monitored by accelerometer and heart rate sensors implemented on wearables or, in some cases, on smartphones. Nowadays, also global positioning systems are often used for physical and exercise activity tracking [42]. The added value of technology is not confined to monitoring patients' PA objectively, but it extends to patients' stimulation and engagement to increase adherence [43]. For instance, human interface technology can reduce the gap between therapists and patients, who may feel more responsible to adhere when directly supervised [44]. Furthermore, technology tools offer the possibility for a more enjoyable and motivational approach to PA programs (e.g., exergaming) [44, 45]. Taken together, this evidence suggests the high potential of the monitoring and userinterface technology in promoting an active lifestyle in persons with disabilities and chronic diseases.

The aim of this systematic review was to evaluate whether in patients with chronic diseases experiencing fatigue the use of monitoring technology would improve adherence to a PA program in the mHealth and eHealth space such as a home and/or rehabilitation settings.

\section{Methods}

This systematic review was conducted following PRISMA's guidelines [46], and it was registered in the Prospero Database (CRD42018109081). The research question for this systematic review was: "Does monitoring technology affect adherence to physical activity and exercise programs in patients with chronic diseases perceiving fatigue?". Studies published in English from the year 2006 were included in the search. We did not exclude conference abstracts and requested full-texts when they were not available. We collected studies from Embase, Medline, and Biosis on the $19^{\text {th }}$ of March 2018, using this keywords $(((()($ physical $n 1$

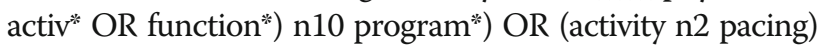
OR rehabilitation) AND (adhere OR motiv* OR complai$\left.\mathrm{n}^{*}\right)$ )AND (pedometer* OR accelerometer* OR monitor* OR wearable* OR watch* OR phone* OR band* OR heart rate* OR monitor* OR telemonitor* OR mhealth* OR ehealth* OR telehealth*)) AND ((chronic* n5 disease* OR ill*) OR copd OR fatigue OR diabetes OR heart failure OR cancer OR malignant neoplasm OR osteoarthritis OR rheumatoid arthritis OR fibromyalgia OR cancer fatigue OR chronic fatigue syndrome)))). An additional search in the same databases was executed on the $23^{\text {rd }}$ of March 2018 adding "cancer" to the previous search. The search was executed using Scientific \& Technical Information Network library; additionally, three more searches were conducted with the same key words in the same database as mentioned above with the inclusion of Scopus, and SPORTDiscus the $30^{\text {th }}$ of April 2019.

In order to be included, the studies had to satisfy the following criteria: Was this study conducted in a clinical population experiencing fatigue? Was monitoring technology used? Did this study concern activity/exercise programs? Was adherence calculated or derivable from this study? Was monitoring technology used to improve adherence? The use of monitoring technology in improving adherence referred to continuous and long at least one month period. Studies in which monitoring technology was used, but not to provide any feedback, were excluded. For instance, studies in which participants were blinded to pedometers or accelerometers output were not included. Full text screening was conducted by two independent researchers (A.A. and F.S.).

Adherence was generally defined as PA performed over the total PA target prescribed times 100. If adherence was not directly available, we calculated it.

Quality was assessed according to the Downs and Black checklist [47] cited in the Cochrane's Handbook for Systematic Reviews of Interventions (see Table 1) [48]. We assigned a score of 1 in case of a positive answer, 0 when negative or unknown. Studies with a total score from 11 to 15 were considered "high quality", 610 "medium quality", 0-5 "low quality." Two studies scored high quality $[49,50]$, the other studies were classified as medium quality (Table 2 ).

\section{Results}

The study search process is presented in the Fig. 1. The search identified 1790 records, 1674 after removing duplicates. During the process of study selection and data analysis, eight more studies were identified through reference lists as possibly interesting and included. From records screened, we selected 312 abstracts. A total of 85 full-text manuscripts were reviewed. In the end, eight studies were selected based on our inclusion criteria and included in this systematic review. Two studies referred to the same data collection $[44,50]$. Main outcomes of included studies are summarized in Table 3.

\section{Patient Groups}

This systematic review includes eight studies, comprising 195 patients, 89 COPD [41, 45, 46, 48, 51], 39 cancer 
Table 1 Quality assessment questions

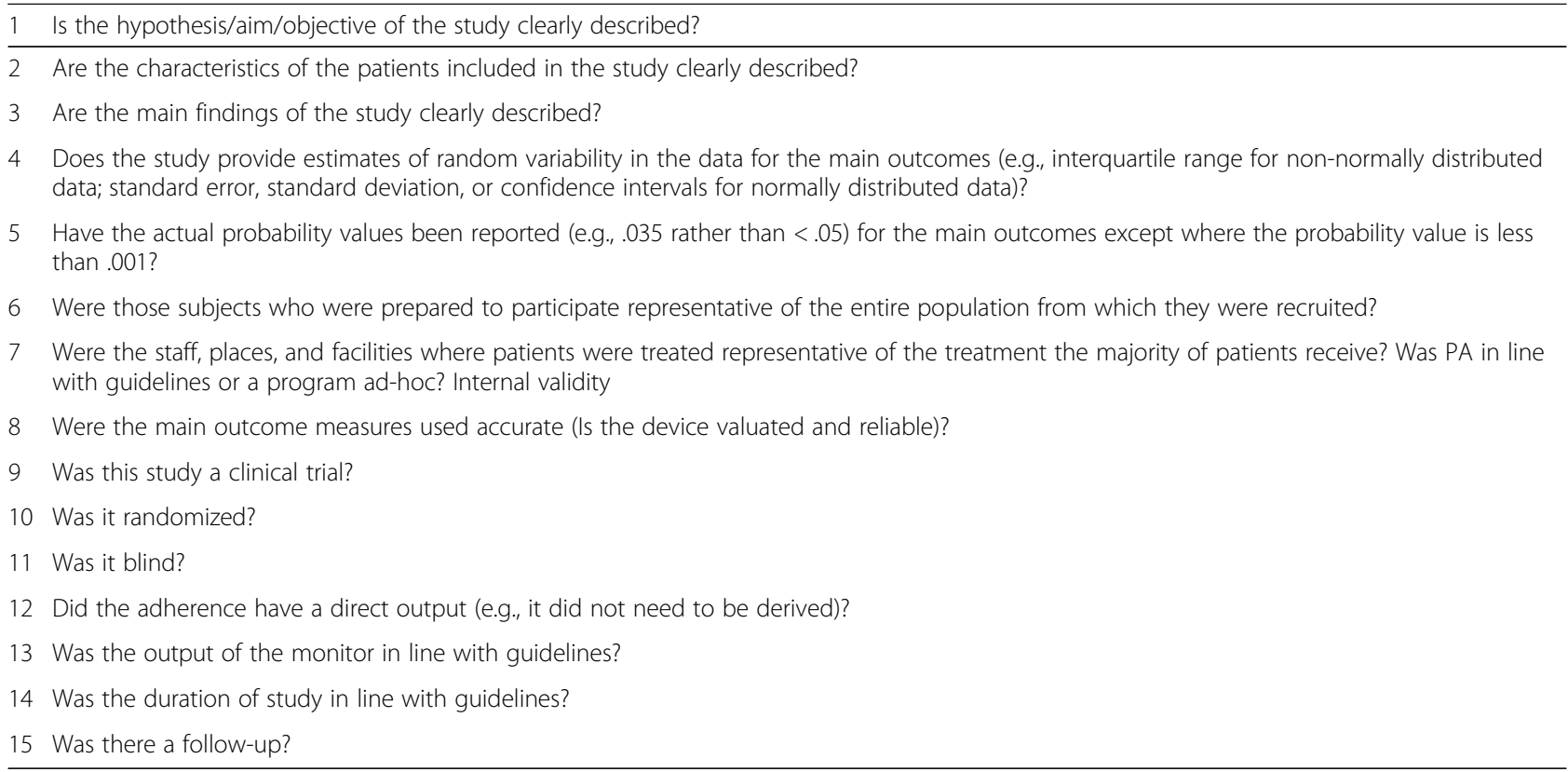

patients [51], 43 cancer survivors [55], and 24 patients with HF [53]. Mendoza et al. recruited COPD patients with both I, II, III, and IV GOLD 2011 [56] classification [49]. The same patients had no $(n=8)$, slight $(n=22)$, moderate $(n=14)$, and severe $(n=8)$ dyspnea and none had very severe dyspnea, assessed with the Modified Medical Research Council (MMRC) dyspnea scale [49].
Patients with other chronic conditions were excluded [49]. Moy et al. recruited patients with no $(n=4)$, slight $(n=10)$, moderate $(n=6)$, severe $(n=4)$, and very severe $(n=1)$ dyspnea assessed with the MMRC scale [54]. Benzo et al. recruited patients with II, III, and IV COPD GOLD 2011 [56] stage [52]. The description of the ten COPD patients undergoing 2 years intervention

Table 2 Quality assessment and risk of bias

\begin{tabular}{|c|c|c|c|c|c|c|c|c|}
\hline $\begin{array}{l}\text { Study } \\
\text { question }\end{array}$ & $\begin{array}{l}\text { Backman et al. } \\
\text { [51] }\end{array}$ & $\begin{array}{l}\text { Benzo et al. } \\
\text { [52] }\end{array}$ & $\begin{array}{l}\text { Gary et al. } \\
\text { [53] }\end{array}$ & $\begin{array}{l}\text { Hoaas et al. } \\
\text { [44] }\end{array}$ & $\begin{array}{l}\text { Hoaas et al. } \\
\text { [50] }\end{array}$ & $\begin{array}{l}\text { Mendoza et al. } \\
\text { [49] }\end{array}$ & $\begin{array}{l}\text { Moy et al } \\
\text { [54] }\end{array}$ & $\begin{array}{l}\text { Pinto et al. } \\
{[55]}\end{array}$ \\
\hline 1 & 1 & 1 & 1 & 1 & 1 & 1 & 1 & 1 \\
\hline 2 & 1 & 0 & 1 & 0 & 1 & 1 & 1 & 1 \\
\hline 3 & 1 & 1 & 1 & 1 & 1 & 1 & 1 & 1 \\
\hline 4 & 0 & 0 & 1 & 0 & 1 & 1 & 1 & 1 \\
\hline 5 & 0 & 0 & 1 & 0 & 1 & 1 & 1 & 1 \\
\hline 6 & 0 & 0 & 0 & 0 & 0 & 0 & 0 & 0 \\
\hline 7 & 0 & 0 & 0 & 0 & 0 & 0 & 0 & 0 \\
\hline 8 & 1 & 1 & 0 & 0 & 1 & 1 & 1 & 1 \\
\hline 9 & 0 & 1 & 0 & 1 & 1 & 1 & 1 & 0 \\
\hline 10 & 1 & 0 & 1 & 0 & 0 & 1 & 0 & 1 \\
\hline 11 & 0 & 0 & 0 & 0 & 0 & 1 & 0 & 0 \\
\hline 12 & 1 & 1 & 1 & 1 & 1 & 0 & 0 & 1 \\
\hline 13 & 1 & 1 & 1 & 1 & 1 & 1 & 1 & 1 \\
\hline 14 & 0 & 0 & 1 & 1 & 1 & 1 & 0 & 1 \\
\hline 15 & 0 & 0 & 1 & 1 & 1 & 0 & 0 & 0 \\
\hline Total & 7 & 6 & 10 & 7 & 11 & 11 & 8 & 10 \\
\hline Score & Medium & Medium & Medium & Medium & High & High & Medium & Medium \\
\hline
\end{tabular}




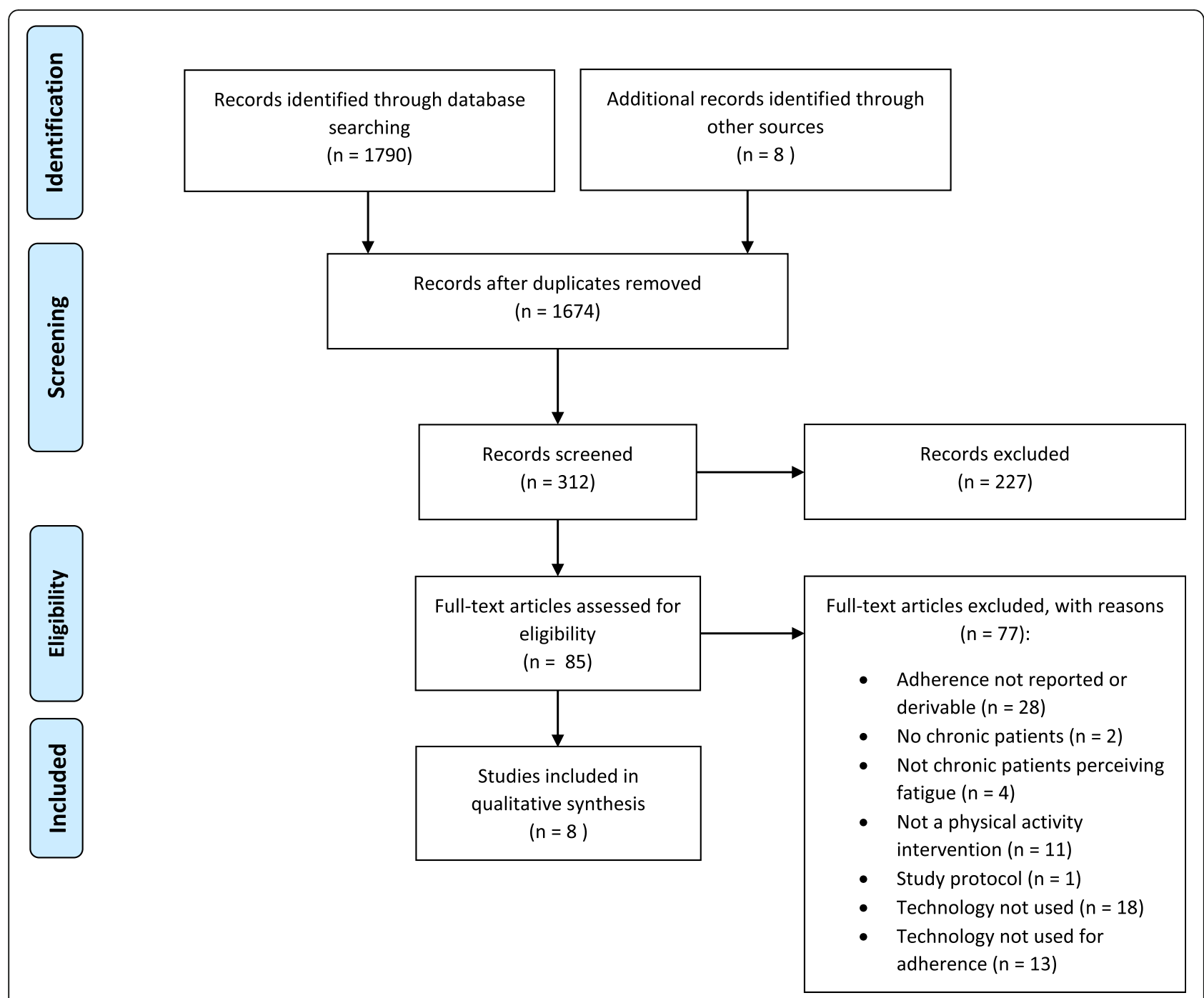

Fig. 1 Selection process of studies

and one additional year of unsupervised follow-up [44, 50] is presented in Zanaboni et al. [57], showing that the majority of them had high COPD assessment test (CAT) scores $(n=6)$, and the rest medium $(n=3)$ or low $(n=1)$ CAT scores. Backman el al. recruited patients with breast and colorectal cancer (CRC), all of whom undergoing chemotherapy treatment [51]. Pinto et al. recruited 43 breast cancer survivors, with cancer at stage $0(n=8), 1(n=$ $17)$, and $2(n=18) ; 55.8 \%$ of them received chemotherapy [55]. Gray et al. selected patients with stable systolic HF, with slight and marked limitation to PA, class II and III of the New York Association (NYHA) classification [53].

\section{Program Characteristics}

In three studies, PA programs were based on step counts $[49,51,54]$; in other studies, the goal was expressed in minutes of activity $[44,50,52,53,55]$. PA programs were personalized and activity levels adjusted in four studies [49, 53-55], with the final goal to meet the guidelines formulated in the different studies. Hoaas et al. prescribed a home-based interval training based on heart rate, $4 \times 4 \mathrm{~min}$ of intense walking, three times per week [44, 50]. Backman et al. proposed 10,000 steps per day as a fixed goal [51]. Benzo et al. used $12 \mathrm{~min}$ of slow walking in a COPD home-based rehabilitation program, 6 days/week [52]. In Backman et al., PA increased in the intervention group compared with the control group, but decreased during the study [51]. Benzo et al. did not analyze PA as pre-post intervention [52]. Programs lasted a minimum of 8 weeks to a maximum of 2 years.

\section{Monitoring Technology Characteristics}

Pedometers were the prevalent type of monitoring technology used $[49,51,54,55]$; other studies used activity monitors [52], pulse oximeters [44, 50], and heart monitors [53]. For seven out of eight studies, it was possible 


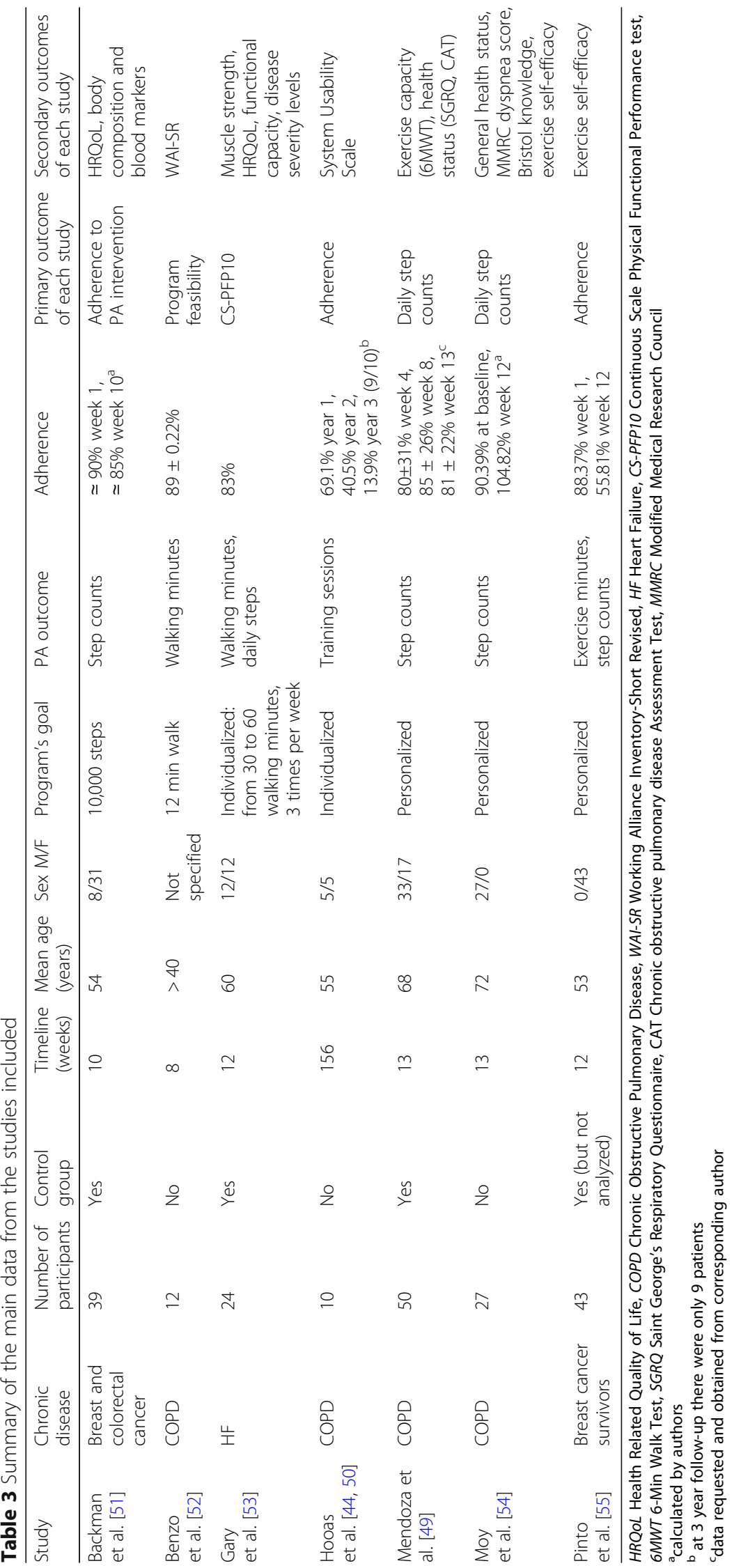


to determine the type of sensing technology used. The sensors were a 2-axial [53] and 3-axial accelerometer [49], a pair of piezoelectric sensors oriented at $90^{\circ}$ [54], either a spring-levered or a piezo-electric pedometer [55], and finally an unspecified accelerometer [52]. The patients could check the last 7 days steps in all four pedometers, which kept around 30-40 days in memory. The activity monitor could store up to 21 days. Hoaas et al. [44] used a portable finger pulse oximeter (Nonin GO2 LED) to measure $\mathrm{SPO}_{2}$ and heart rate during exercise. This device does not have memory, thus patients were asked to write down their maximal HR, that they observed during every exercise session [57]. In Gary et al. in addition to the pedometer, a heart rate chest strap (Polar) was used [53]. Two devices had USB connection $[51,54]$, Vivofit 2 used in Benzo et al. had Bluetooth connectivity [52]; the DigiWalker, Omron HJ-112, the Tanita PD-724, and the Nonin GO2 LED did not seem to have any type of digital connectivity $[49,53,55,57]$. Battery life ranged from 6 months to 1 year. All devices seemed to have a rather reasonable price, not exceeding $£ 60$ per unit (£24 to $£ 58$ ). Except for Hoaas et al. [44, 50] in which a $£ 80$ pulse oximeter, a $£ 400$ tablet, and a $£ 600$ treadmill were used per patient, making it up to a total of $£ 1085$ per patient in equipment cost only [57]. Two studies implemented PA monitoring on a website connected to the pedometers $[52,54]$. PA monitoring was continuous for all studies.

\section{Monitoring Technology Usability}

In Mendoza et al. and Backman et al., step counts measured by pedometers were manually reported in daily diaries [40, 52]. In contrast, in Benzo et al. and Moy et al., devices (i.e., pedometers and activity monitor armbands) were connected directly to an internet-supported digital system, so that patients could monitor their progress online [42, 53]. In Hoaas et al. [44, 50], videoconferences were used to remotely supervise training sessions, as the tablet was fixed onto the treadmill [57], during this conferences HR values were reported. In Gary et al. [53], the HF patients kept training session logs, and these were weekly reviewed by a nurse or an exercise specialist. Patients received monthly, or weekly, motivational support, in the form of calls, messages on a personal webpage, or e-mails in every study [44, 49-53, 55]. Motivational messages were shown to help patients increase their PA levels [58]. Benzo et al. and Backman et al. provided their patients with a fixed goal $[51,52]$. Instead, in other three studies, the researchers gave periodical goals. Mendoza et al. set goals during weekly calls [52], Pinto et al. during monthly appointments [54], and Moy et al. delivered the first goal by e-mail; afterwards, patients could check their weekly goals directly from the website [53]. Hoaas et al.'s $[44,57]$ goals were set by using the rating of perceived exertion scale and the participants were motivated via videoconferencing [57].

Mendoza et al., Moy el al., and Pinto et al. provided personalized goals [52-54]. In these studies, patients' PA increased. In fact, only Backman et al. did not report a PA increase; probably due to patients' sufficiently active baseline levels (9000 steps/day) [40]. Only in Pinto et al. and Gary et al., the program was prescribed using heart rate monitoring to individualize moderate PA [53]. Goals in Gary et al. [53] were based on HR and RPE and progressively increased from 50 to $70 \%$ of their maximum.

Mendoza et al. considered pedometers user-friendly and reported experience of pedometer usability [52]. Conversely, Backman et al. observed that their patients experienced difficulties to manually record steps in activity diaries [40]. Moy et al. reported some problems in using pedometers and a website [53]. The main two problems were pedometer wearability at the waist, and first step counts upload; nonetheless, these difficulties were resolved in time.

\section{Fatigue}

Of the selected studies, only Backman et al. quantified fatigue, assessed by means of the European Organization for Research and Treatment of Cancer quality of Life Questionnaire (EORTC QLQ-C30) [51]. Fatigue did not significantly increase during the intervention in this study and no differences were found compared to control group [51]. Although the other studies did not directly quantify fatigue, the patient populations investigated are known to suffer from fatigue, which exacerbates during exercise. Fatigue is in fact well characterized in all diseases included in this systematic review [59-62].

\section{Adherence Outcomes}

Only two studies reported adherence as percentage of the total goal $[51,55]$. Three reported it as the participation to PA session out of the total recommendation $[44,50,53]$. In the other studies, we derived adherence as follows. Mendoza et al. [49] provided us the data to calculate adherence. Then, we applied a one-way analysis of variance (ANOVA) for repeated measures to evaluate adherence values of the intervention group over time. Adherence values did not statistically differ. In the remaining studies $[52,54]$, adherence was calculated by us as a ratio between the executed PA and the PA goal (for details see adherence column Table 3).

\section{Discussion}

The purpose of this systematic review was to evaluate the potential of using monitoring technology to improve adherence to a PA program, for patients with chronic diseases experiencing fatigue complaints. When looking at qualitative evidence provided by this systematic review, we concluded that it was not possible to establish 
whether the use of monitoring technology was able to positively influence physical activity adherence in patients with chronic diseases experiencing fatigue.

From 1790 hits, we included only eight studies. We selected the studies in which monitoring technology was used by patients to control their PA level and their progress and by doing so this would potentially improve program adherence. When defining the effect of monitoring technology to improve physical activity adherence, we included any technological tools which provided direct feedback to users (e.g., progress, achievements), as such able to increase their awareness and/or engagement, ultimately possibly resulting in a greater program adherence. We included only studies with patients with chronic diseases to whom fatigue could represent a serious obstacle to being active.

\section{Monitoring Technology Tools}

The results underline that pedometers are the most used monitoring technology tools in the clinical context. The studies included in our systematic review seem to confirm that pedometers help to increase PA [63]. Unfortunately, an increase in PA was not always followed by health outcome improvements [40]. Although defining a PA program goal in steps is straightforward for most patients, this may in some cases not elicit a clear increase in their cardiorespiratory fitness. Moreover, half of the participants who reached a 10,000 steps target did not meet the minimum goal of $30 \mathrm{~min}$ of daily activity (in 10-min bouts) [64]. Programs that prescribed exercise intensity improved cardiorespiratory fitness more than programs prescribing only quantity of exercise (e.g., steps) [65]. Despite some difficulties, it could be inferred that the use of wearable devices, such as pedometers, is convenient and user-friendly for patients with chronic diseases even when they are elderly. However, it needs to be acknowledged that the accuracy of step counting at lower speeds has been found to be reduced $[66,67]$. The only long-term adherence intervention study had, however, a small sample size $(n=10)$, and it did show a drastic decrease in adherence when the training sessions were no longer remotely supervised $[44,50]$. This systematic review seems to point toward the idea that monitoring technology can help to reduce the gap between patients and therapists. Furthermore, it confirms the importance, and often the necessity, of maintaining a personal relationship through calls or personalized motivational messages. If these messages are impersonal and automated, this can lead to a lower efficacy [68].

\section{Drop-Outs and Fatigue}

In Mendoza et al., drop-out rate was 4.9\% (5 COPD patients, three in the intervention group and two in the control group). No specific causes were reported. Moy et al. described that three COPD patients did not complete the study (11\%), reporting medical problems. Further, two patients were excluded from data analysis for incomplete step uploading, and interruptions due to medical problems [54]. Pinto et al. included the low rate of attrition (5\%) as one of the strengths of their study [55]. In Backman et al., $26 \%$ of the cancer patients dropped-out, because of personal reasons, stress, treatments side effects, and fatigue [51]. For these patients, stress during treatment, health reasons, and fatigue constituted the barriers to nonparticipation. Despite the burdensome treatments, cancer patients showed a good or optimum PA level at the end of the interventions ( $\simeq 8500$ and 14,500 steps/day) $[51,55]$. Indeed often, cancer patients did not have problems to complete PA interventions [69]. No dropouts were recorded by Gary et al. [53] during the intervention $(n=$ 24) in HF patients. However, the screening of 615 potential participants led to a $75 \%$ rejection rate (documented non-adherence to medication was one of the exclusion criteria), and of the $25 \%$ finally invited only $4 \%$ responded to the invitation, enrolling a well-selected sample. Only one of the ten COPD patients in the study of Hoaas et al. $[44,50]$ dropped out during the 3-year study period.

An important selection criterion for this systematic review was the presence of fatigue suffered by the patients with chronic diseases undergoing a PA program. Although fatigue was known to be present in all eight studies selected, only Backman et al. assessed fatigue directly [51]. Fatigue was one of most relevant symptoms both for the intervention and the control group. Fatigue did not change during the study, yet according to Backman et al., it could be a possible cause of decrement in adherence [51].

PA levels of COPD patients were lower compared to cancer patients, yet the age of the COPD patients was higher compared to the other studies included in this systematic review and COPD conditions were moderate. This systematic review confirmed also that walking is the most common and feasible way to approach physical activity for patients with chronic diseases; in particular for cancer patients [69]. Only in Backman et al., colorectal cancer patients reported barriers to walking, as their therapy's complications included hand-foot syndrome [51].

\section{Self-Efficacy and Intervention Duration}

The importance of self-efficacy of patients with chronic diseases in carrying out a PA program is known [70]. In fact, Pinto et al. showed how self-efficacy values represent a predictor of goal achievement [55]. Interestingly, Moy et al. looked at self-efficacy at the beginning and at the end of the study and found no changes, whereas PA level increased [54]. Also in Hoaas et al. [44, 50], self-efficacy did not change from 2 to 3 years, while adherence dropped drastically. Backman et al. underlined how the length of the intervention influences adherence to a PA 
program; the longer the study, the lower the adherence [51]. Yet, Hoaas et al. outcomes seemed to suggest that cessation of remote supervision of training sessions was the underlying cause of the drop in adherence. The mean duration of the interventions considered in these studies was 32 weeks, ranging from 8 to 156 weeks.

\section{Clinical Outcomes}

Backman et al. showed that cancer patients with a 10,000 steps goal for 10 weeks and adherence levels of $85 \%$, decreased body weight without a change in body composition and decreased blood pressure, but did not show changes in inflammatory markers. Moreover, those cancer patients reported a decrease in symptoms but no alterations in QoL [51]. Moy et al. observed that COPD patients, undergoing 13 weeks personalized PA program with a very high adherence, did not change their general health status (measured with Medical Study Short Form36 questionnaire) and neither their dyspnea [54]. Conversely, Mendoza et al. [49] with a similar program in similar patients as Moy et al. [54], but lower adherence, found changes in the health status (SGRQ and CAT) but not in dyspnea (MMRC). Yet in Mendoza et al. [49], absolute step counts were higher than in Moy et al. [54] (from 4000 to 7000 steps circa). Gary et al. [53] found a large increase in QoL (i.e., 23 point on the Minnesota Living With Heart Failure Questionnaire) in the intervention group, which had $83 \%$ adherence, versus no change in the control group. Hoaas et al. $[44,50]$ did not observe significant clinical changes in 2 years intervention but adherence after the first year was already below $50 \%$. Benzo et al. and Pinto et al. did not report clinical outcomes and/or health status parameters $[52,55]$.

\section{Limitations}

This systematic review has several limitations. One of the main limitations is the lack of adherence measurements the included literature analyzed. Moreover, there is poor consistency on what the term adherence really means when referring to PA programs. Pinto et al. and Backman et al. considered adherence both as continuous (minutes of exercise) and dichotomous (if participants met their goal) outcomes [51, 55]. Benzo et al., Gary et al., and Hoaas et al., reported adherence as a percentage of number of sessions performed, assuming that each daily session begun was completed $[44,50,52]$. The other two did not define adherence, which was calculated by us as the weekly mean of daily scores expressed as a percentage of the daily goal.

The low number of studies, the small sample size, the overall short duration of interventions ( $\max 13$ weeks), with the exception of one study, and the low heterogeneity of chronic disease types (only COPD, HF, and cancer patients) represent further limitations. At the same time, these limitations identify gaps in current literature regarding using wearable technology to stimulate an active lifestyle in special populations, based on which recommendations for future research directions can be determined. Finally, although this systematic review focused on patients with chronic diseases experiencing fatigue, fatigue was explicitly measured only in one study of the eight included. We have selected studies where, based on the literature, it was safe to assume the presence of fatigue complaints.

\section{Conclusions}

The small body of evidence found in this systematic review does not allow us to establish whether the use of wearable technology was able to improve adherence to PA programs in patients with chronic diseases experiencing fatigue. The eight studies finally selected were of mediumhigh quality but with small sample sizes and including only three types of chronic diseases. In general, the studies analyzed in this systematic review showed high levels of adherence associated with monitoring technology, yet for rather short PA programs ( $\max 13$ weeks). Indeed the longest study taken into account, 2 years intervention plus 1 year follow-up showed a drop in adherence from $70 \%$ at the end of year 1 down to $40 \%$ at the end of year 2 [44]. Furthermore, in these studies, there did not seem to always be a strong relation between high adherence to PA programs and positive clinical health outcomes. This review has also underlined the necessity to clarify and standardize the definition of adherence. Six out of eight studies used very similar monitoring technology, predominantly pedometers, and all were worn at the waist. The adoption of mobile applications to monitor adherence in this population was still absent. Although these patients with chronic diseases experienced fatigue, this did not seem to influence adherence levels. Technology was mainly used to objectively monitor PA, rather than to improve adherence. Yet, the use of monitoring technology to improve fitness and wellbeing is a clear trend and may potentially be particularly useful as assistive tool to stimulate an active lifestyle and exercise adherence in special populations. Future research should investigate whether monitoring technology is effective in improving PA adherence, and if so, how effective it is and what the best implementation would be.

\section{Abbreviations}

6MWT: 6-Minutes walking test; ACSM: American College of Sports Medicine; ANOVA: Analysis of variance; CAT: Chronic obstructive pulmonary disease assessment test; COPD: Chronic obstructive pulmonary disease; CS-

PFP10: Continuous scale physical functional performance test; HF: Heart failure; HR: Heart rate; HRQoL: Health-related quality of life; MMRC: Modified Medical Research Council; PA: Physical activity; QoL: Quality of life; SGRQ: Saint George's Respiratory Questionnaire; T2DM: Type 2 diabetes mellitus; WAI-SR: Working Alliance Inventory-Short Revised 


\section{Acknowledgements}

Not applicable.

\section{Availability of Data and Material}

Not applicable.

\section{Authors' Contributions}

$A A, F S$, and, FJH have set the keywords for the literature search. AA and FS have run the literature search and analyzed the results. AA and FS have draft the first version of the manuscript. FJH, MB, and, ALT have reviewed and improved the manuscript. All authors read and approved the final manuscript.

\section{Funding}

The funder provided support in the form of salary to F.S., but did not have any additional role in the study design, data collection, analysis, and preparation of the manuscript. All other authors did not receive any funding for this study.

\section{Ethics Approval and Consent to Participate}

Not applicable.

\section{Consent for Publication}

Not applicable.

\section{Competing Interests}

Andrea Albergoni was doing an internship at Philips Electronics B.V. during his contribution in this work. Florentina J. Hettinga, Matteo Bonato, and Antonio La Torre do not have any conflict of interests. Francesco Sartor works at Philips Electronics B.V.

\section{Author details}

'Department of Biomedical Sciences for Health, Università degli Studi di Milano, Milan, Italy. ${ }^{2}$ Department of Patient Care \& Measurements, Philips Research, Eindhoven, The Netherlands. ${ }^{3}$ School of Sport Rehabilitation and Exercise Sciences, University of Essex, Colchester, UK. ${ }^{4}$ Department of Sport, Exercise and Rehabilitation, Northumbria University, Newcastle, UK. ${ }^{5}$ RCCS Istituto Ortopedico Galeazzi, Milan, Italy. ${ }^{6}$ College of Health \& Behavioural Science, Bangor University, Bangor, UK. ${ }^{7}$ Philips Electronics Nederland B.V, HTC 34 1.011, P.O. Box WB61, 5656, AE, Eindhoven, The Netherlands.

Received: 23 January 2019 Accepted: 19 August 2019

Published online: 12 September 2019

\section{References}

1. Durstine, J. Larry; Moore Geoffrey E; Painter, Patricia L.: Roberts, Scott OR ACSM's exercise management for person with chronic disease and disabilities. 3rd ed. American College of Sport Medicine. 2009.

2. Pedersen BK, Saltin B. Exercise as medicine-evidence for prescribing exercise as therapy in 26 different chronic diseases. Scand J Med Sci Sport. 2015:25:1-72.

3. Smith B, Kirby N, Skinner B, Wightman L, Lucas R, Foster C. Infographic Physical activity for disabled adults. Br J Sports Med. 2018;53(6):335-6.

4. Piepoli MF. Exercise training in heart failure. Curr Cardiol Rep. 2005;7:216-22

5. Teixeira-Lemos E, Nunes S, Teixeira F, Reis F. Regular physical exercise training assists in preventing type 2 diabetes development: Focus on its antioxidant and anti-inflammatory properties. Cardiovasc. Diabetol. 2011;10:12.

6. Aune D, Norat T, Leiitzman M, Tonstad SVL. Physical activity and the risk of type 2 diabetes: a systematic review and dose response meta-analysis. Eur $J$ Epidemiol. 2015;30:529-42.

7. Jones SB, Thomas GA, Hesselsweet SD, Alvarez-Reeves M, Yu H, Irwin ML Effect of exercise on markers of inflammation in breast cancer survivors: The yale exercise and survivorship study. Cancer Prev Res. 2013;6(2):109-18.

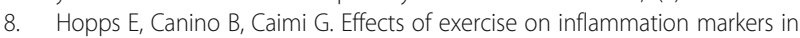
type 2 diabetic subjects. Acta Diabetol. 2011;48:183-9.

9. Garcia DO, Thomson CA. Physical activity and cancer survivorship. Nutr Clin Pract. 2014:29:768-79.

10. Hartman JE, Boezen HM, De Greef MHG, Bossenbroek L, Ten Hacken NHT. Consequences of physical inactivity in chronic obstructive pulmonary disease. Expert Rev. Respir. Med. 2010;4:735-45.
11. Palmefors H, DuttaRoy S, Rundqvist B, Börjesson M. The effect of physical activity or exercise on key biomarkers in atherosclerosis - A systematic review. Atherosclerosis. 2014;235:150-61.

12. Segura-Jiménez V, Estévez-López F, Castro-Piñero J, Álvarez-Gallardo IC, Soriano-Maldonado A, Borges-Cosic M, et al. Association of Patterns of moderate-to-vigorous physical activity bouts with pain, physical fatigue, and disease severity in women with fibromyalgia: the al-Ándalus Project. Arch Phys Med Rehabil. 2019.

13. Chambers D, Bagnall A-M, Hempel S, Forbes C. Interventions for the treatment, management and rehabilitation of patients with chronic fatigue syndrome/ myalgic encephalomyelitis: an updated systematic review. J R Soc Med. 2006;99:506-20.

14. Taylor RS, Walker S, Smart NA, Piepoli MF, Warren FC, Ciani O, et al. Impact of exercise rehabilitation on Exercise capacity and quality-of-life in heart failure. J Am Coll Cardiol. 2019;73:1430-43.

15. Richardson CR, Faulkner G, McDevitt J, Skrinar GS, Hutchinson DS, Piette JD. Integrating physical activity into mental health services for persons with serious mental illness. Psychiatr Serv. 2005;56:324-31.

16. Dairo YM, Collett J, Dawes H, Oskrochi GR. Physical activity levels in adults with intellectual disabilities: a systematic review. Prev Med Reports. 2016:4:209-19.

17. Jefferis BJ, Sartini C, Lee IM, Choi M, Amuzu A, Gutierrez C, et al. Adherence to physical activity guidelines in older adults, using objectively measured physical activity in a population-based study. BMC Public Health. 2014;14:382.

18. Troiano RP, Berrigan D, Dodd KW, Mâsse LC, Tilert T, Mcdowell M. Physical activity in the United States measured by accelerometer. Med Sci Sports Exerc. 2008:40:181-8

19. Chao D, Foy CG, Farmer D. Exercise adherence among older adults. Control Clin Trials. 2000;21:S212-7.

20. Jack K, McLean SM, Moffett JK, Gardiner E. Barriers to treatment adherence in physiotherapy outpatient clinics: A systematic review. Man Ther. 2010;15:220-8.

21. Craike M, Gaskin CJ, Courneya KS, Fraser SF, Salmon J, Owen PJ, et al. Predictors of adherence to a 12-week exercise program among men treated for prostate cancer: ENGAGE study. Cancer Med. 2016;5:787-94.

22. Radbruch L, Strasser F, Elsner F, Gonçalves JF, Løge J, Kaasa S, et al. Fatigue in palliative care patients—an EAPC approach. Palliat Med 2008;22:13-32.

23. Hannan M, Bronas UG. Barriers to exercise for patients with renal disease: an integrative review. J. Nephrol. 2017;30(6):729-41.

24. Andersson M, Stridsman C, Rönmark E, Lindberg A, Emtner M. Physical activity and fatigue in chronic obstructive pulmonary disease-a population based study. Respir Med 2015;109:1048-1057.

25. Bower JE. Cancer-related fatigue-mechanisms, risk factors, and treatments. Nat Rev Clin Oncol. 2014;11:597-609.

26. Kalra S, Sahay R. Diabetes fatigue syndrome. Diabetes Ther. Springer. 2018;9:1421-9.

27. Fritschi C, Quinn L. Fatigue in patients with diabetes: a review. J Psychosom Res. 2010;69:33-41.

28. Nilsson KR, Duscha BD, Hranitzky PM, Kraus WE. Chronic heart failure and exercise intolerance: the hemodynamic paradox. Curr. Cardiol. Rev. 2008;4(2):92-100.

29. Abonie US, Sandercock GRH, Heesterbeek M, Hettinga FJ. Disability and rehabilitation effects of activity pacing in patients with chronic conditions associated with fatigue complaints: a meta-analysis. Disabil Rehabil. 2018:1-10.

30. Tuso P. Strategies to increase physical activity. Perm J. 2016;19:84-8

31. Avery L, Flynn D, Dombrowski SU, van Wersch A, Sniehotta FF, Trenell MI. Successful behavioural strategies to increase physical activity and improve glucose control in adults with type 2 diabetes. Diabet Med. 2015;32:1058-62.

32. Sallis R, Franklin B, Joy L, Ross R, Sabgir D, Stone J. Strategies for promoting physical activity in clinical practice. Prog Cardiovasc Dis. 2015;57:375-86.

33. Alingh RA, Hoekstra F, Van Der Schans CP, Hettinga FJ, Dekker R, Van Der Woude LH. Protocol of a longitudinal cohort study on physical activity behaviour in physically disabled patients participating in a rehabilitation counselling programme: ReSpAct. Open. 2015;5:7591.

34. Beinart NA, Goodchild CE, Weinman JA, Ayis S, Godfrey EL. Individual and intervention-related factors associated with adherence to home exercise in chronic low back pain: a systematic review. Spine J. 2013;13:1940-50.

35. Tucker JM, Welk GJ, Beyler NK. Physical activity in U.S. adults: compliance with the physical activity guidelines for Americans. Am J Prev Med. 2011;40:454-61.

36. Poole ES. HCl and mobile health interventions. Transl Behav Med. 2013;3:402-5.

37. Muntaner A, Vidal-Conti J, Palou P. Increasing physical activity through mobile device interventions: a systematic review. Health Informatics J. 2016;22:451-69.

38. Mateo GF, Granado-Font E, Ferré-Grau C, Montaña-Carreras X. Mobile phone apps to promote weight loss and increase physical activity: a systematic review and meta-analysis. J. Med. Internet Res. 2015;17:e253. 
39. Gal R, May AM, van Overmeeren EJ, Simons M, Monninkhof EM. The Effect of physical activity interventions comprising wearables and smartphone applications on physical activity: a systematic review and meta-analysis. Sport Med-Open. 2018;4:42.

40. Qiu S, Cai X, Chen X, Yang B, Sun Z. Step counter use in type 2 diabetes: a meta-analysis of randomized controlled trials. BMC Med. 2014;12:36.

41. Thompson WR. Worldwide survey of fitness trends for 2019. ACSM's Heal Fit J. 2018;22:10-7.

42. Lee JM, Kim Y, Welk GJ. Validity of consumer-based physical activity monitors. Med Sci Sports Exerc. 2014;46:1840-8.

43. Changizi M, Kaveh MH. Effectiveness of the mHealth technology in improvement of healthy behaviors in an elderly population-a systematic review. mHealth. 2017;3:51.

44. Hoaas H, Andreassen HK, Lien LA, Hjalmarsen A, Zanaboni P. Adherence and factors affecting satisfaction in long-term telerehabilitation for patients with chronic obstructive pulmonary disease: A mixed methods study eHealth/ telehealth/ mobile health systems. BMC Med Inform Decis Mak. 2016;16:26.

45. Tough D, Robinson J, Gowling S, Raby P, Dixon J, Harrison SL. The feasibility, acceptability and outcomes of exergaming among individuals with cancer: a systematic review. BMC Cancer. 2018;18:1151.

46. Liberati A, Altman DG, Tetzlaff J, Mulrow C, Gøtzsche PC, loannidis JPA, et al. The PRISMA statement for reporting systematic reviews and meta-analyses of studies that evaluate health care interventions: explanation and elaboration. J Clin Epidemiol. 2009;62:e1-34.

47. Downs SH, Black N. The feasibility of creating a checklist for the assessment of the methodological quality both of randomised and non-randomised studies of health care interventions. J. Epidemiol. Community Health. 1998:52(6):377-84.

48. Higgings JPT, Green S. Cochrane Handbook for Systematic Reviews of Interventions. Cochrane Collab. 2011 Accessed 19 Nov 2018. Available from: www.handbook.cochrane.org

49. Mendoza L, Horta P, Espinoza J, Aguilera M, Balmaceda N, Castro A, et al. Pedometers to enhance physical activity in COPD: a randomised controlled trial. Eur Respir J. 2015;45:347-54.

50. Hoaas H, Morseth B, Holland AE, Zanaboni P. Are physical activity and benefits maintained after long-term telerehabilitation in COPD? Int J Telerehabilitation. 2016;8:39-48.

51. Backman M, Wengström $Y$, Johansson B, Sköldengen I, Börjesson S, Tärnbro $S$, et al. A randomized pilot study with daily walking during adjuvant chemotherapy for patients with breast and colorectal cancer. Acta Oncol (Madr). 2014;53:510-20.

52. Benzo RP, Kramer KM, Hoult JP, Anderson PM, Begue IM, Seifert SJ. Development and feasibility of a home pulmonary rehabilitation program with health coaching. Respir Care. 2017;63(2):131-40.

53. Gary RA, Cress ME, Higgins MK, Smith AL, Dunbar SB. Combined aerobic and resistance exercise program improves task performance in patients with heart failure. Arch Phys Med Rehabil. 2011;92:1371-81.

54. Moy ML, Weston NA, Wilson EJ, Hess ML, Richardson CR. A pilot study of an Internet walking program and pedometer in COPD. Respir Med. 2012;106:1342-50.

55. Pinto BM, Rabin C, Dunsiger S. Home-based exercise among cancer survivors: adherence and its predictors. Psychooncology. 2009;18:369-76.

56. Vestbo J, Hurd SS, Agustí AG, Jones PW, Vogelmeier C, Anzueto A, Barnes PJ, Fabbri LM, Martinez FJ, Nishimura M, Stockley RA, Sin DD, et al. Global strategy for the diagnosis, management, and prevention of chronic obstructive pulmonary disease GOLD executive summary. Am J Respir Crit Care Med. 2013;187:347-65.

57. Zanaboni P, Aarøen Lien L, Hoaas H, Hjalmarsen A, Wootton R. Long-term exercise maintenance in COPD via telerehabilitation: Effects from a 2-year pilot study. 2017;23:74-82

58. Thorup Msn C, Hansen J, Grønkjær M, Andreasen JJ, Nielsen G, Sørensen EE, et al. Cardiac patients' walking activity determined by a step counter in cardiac telerehabilitation: data from the intervention arm of a randomized controlled trial. J Med Internet Res 2016;18.

59. Jones PW, Watz H, Wouters EFM, Cazzola M. COPD: the patient perspective. Int. J. Chron. Obstruct. Pulmon. Dis. 2016:13-20.

60. Evangelista LS, Moser DK, Westlake C, Pike N, Ter-Galstanyan A, Dracup K. Correlates of fatigue in patients with heart failure. Prog Cardiovasc Nurs. 2008;23:12-7.

61. Payne JK, Piper BF, Rabinowitz I, Zimmerman MB. Biomarkers, fatigue, sleep, and depressive symptoms in women with breast cancer: a pilot study. Oncol Nurs Forum. 2006;33:775-83.
62. Vardy JL, Dhillon HM, Pond GR, Renton C, Dodd A, Zhang H, et al. Fatigue in people with localized colorectal cancer who do and do not receive chemotherapy: a longitudinal prospective study. Ann Oncol. 2016;27:1761-7.

63. Bravata DM, Smith-Spangler C, Sundaram V, Gienger AL, Lin N, Lewis R, et al. Using pedometers to increase physical activity and improve health: a systematic review. J. Am. Med. Assoc. 2007;298(19):2296-304.

64. Richardson CR, Newton TL, Abraham JJ, Sen A, Jimbo M, Swartz AM. A meta-analysis of pedometer-based walking interventions and weight loss. Ann. Fam. Med. 2008;6:69-77.

65. Rospo G, Valsecchi V, Bonomi AG, Thomassen IW, van Dantzig S, La Torre A, et al. Cardiorespiratory improvements achieved by American College of Sports Medicine's exercise prescription implemented on a mobile app. JMIR mHealth uHealth. 2016;4:e77.

66. Park W, Lee VJ, Ku B, Tanaka H. Effect of walking speed and placement position interactions in determining the accuracy of various newer pedometers. J Exerc Sci Fit. 2014;12:31-7.

67. Husted HM, Llewellyn TL. The accuracy of pedometers in measuring walking steps on a treadmill in college students. Int J Exerc Sci. 2017;10:146-53.

68. Wang JB, Cadmus-Bertram LA, Natarajan L, White MM, Madanat H, Nichols $J F$, et al. Wearable sensor/device (Fitbit One) and SMS text-messaging prompts to increase physical activity in overweight and obese adults: a randomized controlled trial. Telemed e-Health. 2015;21:782-92.

69. Albrecht TA, Taylor AG. Physical activity in patients with advanced-stage cancer: a systematic review of the literature. Clin. J. Oncol. Nurs. 2012:16:293-300.

70. Gimeno-Santos E, Frei A, Steurer-Stey C, De Batlle J, Rabinovich RA, Raste Y, et al. Determinants and outcomes of physical activity in patients with COPD: A systematic review. Thorax. 2014;69:731-9.

\section{Publisher's Note}

Springer Nature remains neutral with regard to jurisdictional claims in published maps and institutional affiliations.

\section{Submit your manuscript to a SpringerOpen ${ }^{\circ}$ journal and benefit from:}

- Convenient online submission

- Rigorous peer review

- Open access: articles freely available online

High visibility within the field

- Retaining the copyright to your article

Submit your next manuscript at $>$ springeropen.com 